Characterization of the evolution of underwater DBD plasma jet

This article has been downloaded from IOPscience. Please scroll down to see the full text article.

2011 Plasma Sources Sci. Technol. 20034018

(http://iopscience.iop.org/0963-0252/20/3/034018)

View the table of contents for this issue, or go to the journal homepage for more

Download details:

IP Address: 141.211.173.82

The article was downloaded on 06/04/2012 at 16:27

Please note that terms and conditions apply. 


\title{
Characterization of the evolution of underwater DBD plasma jet
}

\author{
John Foster, Bradley Sommers, Brandon Weatherford, Benjamin Yee and \\ Mahima Gupta
}

Department of Nuclear Engineering and Radiological Sciences, University of Michigan, Ann Arbor, MI, USA

Received 26 May 2010, in final form 14 November 2010

Published 3 May 2011

Online at stacks.iop.org/PSST/20/034018

\begin{abstract}
An air plasma jet formed underwater using a coaxial DBD electrode configuration with gas flow is being studied for water treatment applications. The arc-like behavior of the discharge in the absence of any obvious return electrode is not well understood. This study seeks to understand the underlying nature of the arc-like jet mode by studying the evolution of the discharge from microdischarge to jet mode. Photographic and spectroscopic data are used to develop a phenomenological model of discharge evolution. Time-averaged spectra were used to assign an average plume and electron temperature. Calculated jet temperatures were consistent with observed affects such as melting and oxide layer formation on a downstream substrate. The capacity of the microdischarge mode to decompose organic dye in water as a function of time, confirmed previously in the jet mode, was also demonstrated in the absence of the jet.
\end{abstract}

(Some figures in this article are in colour only in the electronic version)

\section{Introduction}

The controlled production of plasma discharges in liquid water has many potential applications ranging from water purification to chemical processing [1-5]. This wide array of applications is attributed to the effect that the plasma has on water reactivity. The plasma discharge drives the production of ozone, $\mathrm{OH}$, localized supercritical water, electrons and excited species that in turn can drive a chemical reaction in the liquid phase $[6,7]$. A plasma discharge in liquid can therefore be classified as an advanced oxidation technique (AOT) [8,9]. To date, the injection of plasma in water essentially revolves around four methods: plasma glow electrolysis where the water acts as an electrode [4], spark or streamer discharges $[10,11]$, pulsed corona discharges [12] and plasma production in injected gas bubbles [13-16]. Those methods involving surface discharges rely on plasma product diffusion from the gas phase into the liquid, and are therefore diffusionlimited and can be expected to have a reduced reaction rate in comparison with direct injection methods such as spark or bubbling. Key to the eventual adoption of any of these discharges for actual practice in water treatment or chemical processing applications is the demonstration of the ability of these methods to process large volumes of liquid $[17,18]$. Discharges that feature in-volume injection or those capable of operating inline with flow are desirable in that they are readily adaptable to industrial processing applications. For example, the ability of the discharge to operate in-volume and inline with the flow of the liquid being processed are key requirements for the treatment of dye-laden process water exhausts generated at textile manufacturing plants [19-21]. If the ultimate goal is to utilize the beneficial attributes of plasmas-in-liquids in industrial settings, then the focus of discharge research must in part address in-volume and inline chemical processing. This follows from the notion that for such discharges to be of practical value, they must be capable of efficiently processing large volumes over time intervals competitive with conventional means; that is, they must address the throughput issue [22].

In earlier work, the operating characteristics of an underwater DBD plasma jet were described [18]. In that work, the plasma jet discussed essentially 'burned' an injected gas cavity formed just downstream of the source when submerged underwater, thereby directly injecting advanced oxidation products and precursors into the water. It was also found that the source was capable of producing its own working vapor underwater without the need for injected airflow. In this case, the discharge was formed and was confined to a steam vapor bubble. The DBD plasma jet is of particular interest in that it is capable of both inline and in-volume operation. 


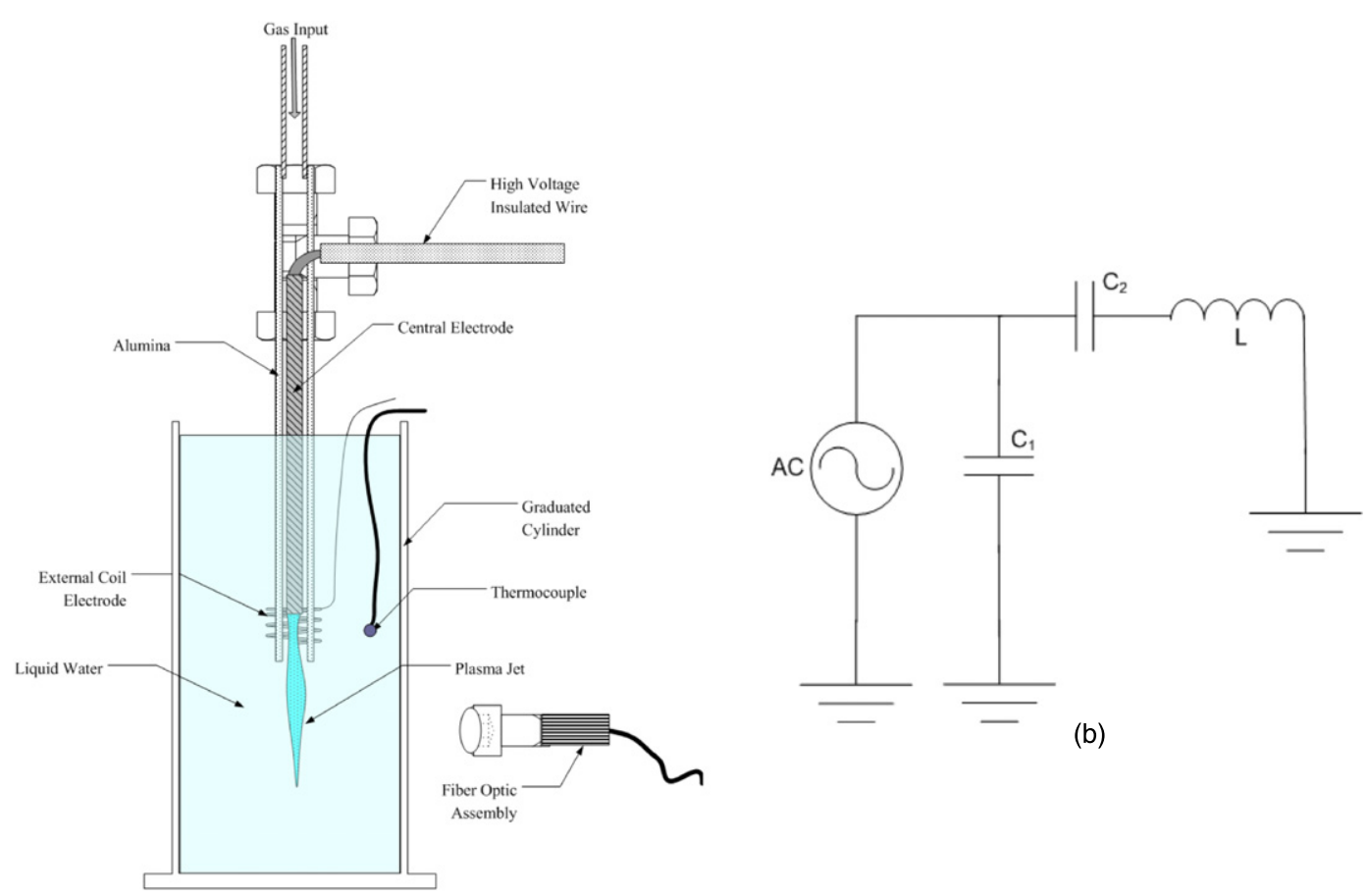

(a)

Figure 1. (a) Schematic depiction of apparatus used to produce plasma jet and characterize its effect on liquid water. $(b)$ Equivalent circuit depiction of apparatus.

As described previously, the discharge behavior, as inferred from the $I-V$ characteristic, behaved more arc-like than a true dielectric barrier discharge. However, an apparent return electrode is missing, suggesting that the jet mode was in fact a manifestation of an intense microdischarge. Charge quenching at the water-jet interface rather than at a solid dielectric is one possible mechanism that could allow an arc-like discharge to be sustained. Here the water interface behaves as a lossy dielectric electrode, thereby giving rise to a larger effective capacitance [23].

In the work discussed herein the evolution of the discharge is further studied elucidating the transition from microdischarge to jet mode using a high-resolution camera. The plasma jet temperature was determined indirectly using optical emission spectroscopy and by assessing the effect of the jet on a downstream substrate. In the previous investigation, it was found that the discharge had the capacity to decompose organic dyes in liquid water. It was demonstrated that jet-derived bulk water temperature increases were not the origin of the decomposition, though the possibility of the production of localized supercritical water at the jet/water interface is still an open question. It was assumed in that work that reactive plasma products created in the jet drove possible dye decoloration. It has been shown by others that plasma formation above a liquid surface can also affect involume chemistry [24]. In the case of the plasma jet, plasma products presumably diffuse directly into the liquid thereby processing the dye. If this is true, then dye decomposition should also be possible by simply 'blowing' the plasmacreated efflux produced by microdischarges in the tube into the liquid water when the source is in microdischarge-mode only. In this present investigation, we also investigated the affect of microdischarge-operation-only on dye concentration as a function of time using a spectrophotometer to access decolorization.

\section{Experimental set up}

A schematic depiction of the apparatus used in this investigation is shown in figure 1. The discharge device consists of a cylindrical, copper electrode coaxially fed into a $5 \mathrm{~mm}$ OD, $2.5 \mathrm{~mm}$ ID dielectric tube. The ground electrode consisted of a coil of tantalum wire wrapped about the end of either an aluminum oxide or a quartz tube. Figure $1(b)$ illustrates the equivalent circuit. Here, $C_{1}$ represents the effective capacitance of the central electrode to ground through the intervening material which consists of the ceramic tubing, the air cavity produced just below the exit plane of the tube during operation and the water. $C_{2}$ represents the capacitance between the central electrode and outer coupling coil, as illustrated in figure 1(a). In this regard, the discharge has the outward appearance of a coaxial dielectric barrier discharge in which the return electrode is immersed in deionized water, which serves as the dielectric. Air was fed into the tubing as depicted in the figure using a compact air compressor. The air compressor flow was measured using an inline air flowrotameter with a readout uncertainty of $\sim 0.1 \mathrm{~L} \mathrm{~min}^{-1}$. In the tests described herein, the discharge tube was submerged into a $100 \mathrm{~mL}$ graduated cylinder containing deionized water with a specific conductance of $0.0714 \mu \mathrm{S} \mathrm{cm}^{-1}$. Figure 2(a) depicts source operation with airflow but no applied voltage. Air injection into the water below the dielectric tube as well as bubble break-off leading to the formation of a multitude of smaller bubbles may be seen in the photograph. In all cases, 


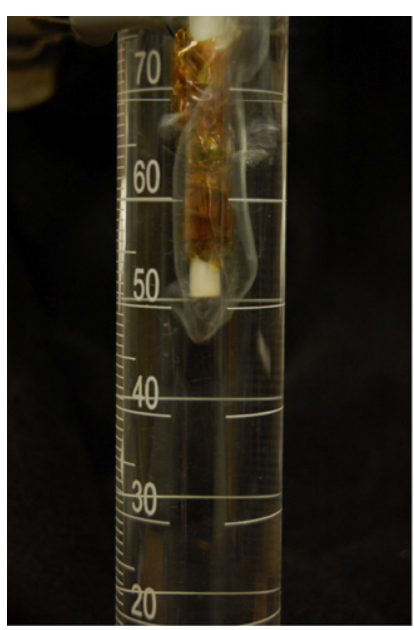

(a)

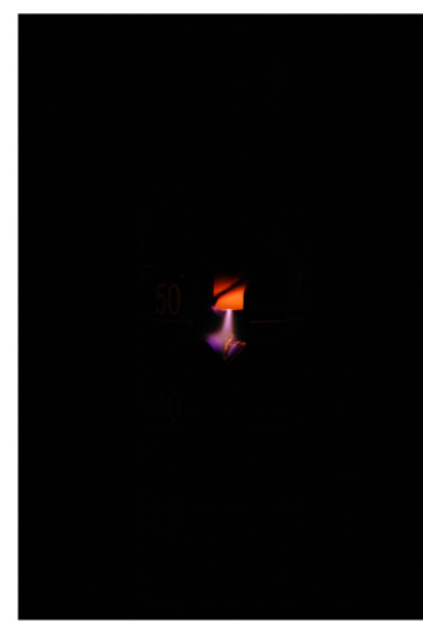

(b)

Figure 2. Photograph of discharge tube with airflow only $(a)$ and with airflow and discharge active $(b)$. Note the plasma jet does not terminate at an external electrode, rather it terminates at the air/water interface. (Discharge: $1 \mathrm{kHz}, 2500 \mathrm{~V}$ amplitude, $1.9 \mathrm{~L} \mathrm{~min}^{-1}$ ).

the injected airflow essentially stagnated after penetrating the water and then turned upward under buoyancy forces as shown in the photograph. This general behavior was unaffected by the presence of the plasma jet. The central electrode was coupled to the variable frequency ac source $(0-200 \mathrm{~V}$ output) through a high voltage transformer $(10 \mathrm{kV}, 1: 50)$, represented simply as the ac source depicted in figure $1(b)$. The operating frequency range investigated in this work was $1-5 \mathrm{kHz}$. Plasma-induced water temperature changes were monitored as a function of time using in situ submerged type $\mathrm{K}$ thermocouples. The current was measured using a Pearson coil rated at up to $120 \mathrm{MHz}$ with a usable rise time of $5 \mathrm{~ns}$. The voltage was measured using a Tektronics $1000: 1$ high voltage probe. The signals from the probes were monitored using a $500 \mathrm{MHz}$ digital oscilloscope, which allowed for the resolution of signal structures down to $10 \mathrm{~ns}$. A photograph of the discharge operating submerged with airflow in deionized water is depicted in figure $2(b)$. Plasma jet length was roughly $1 \mathrm{~cm}$. Orange glow of filtered light through the dielectric tubing, alumina in this case, is also apparent. A spectrophotometer was used to characterize extracted samples of plasma-treated methylene blue (MB) dye samples as described in [18].

Discharge initiation and nominal operation occurred while the discharge tube was submerged under water. Operation was only possible when the source was submerged. During discharge operation an Ocean Optics USB2000 spectrometer with a $25 \mu \mathrm{m}$ slit was used to assess species content as well as estimate electron and heavy particle temperature. The spectrometer's usable range extended from 200 to $1100 \mathrm{~nm}$, making near UV OH lines detectable. Spectrometer resolution is approximately $1.4 \mathrm{~nm}$.

\section{Experimental results}

In order to elucidate the mechanisms behind the operation of the plasma jet, a series of experiments was conducted. These experiments as detailed below investigated (1) the

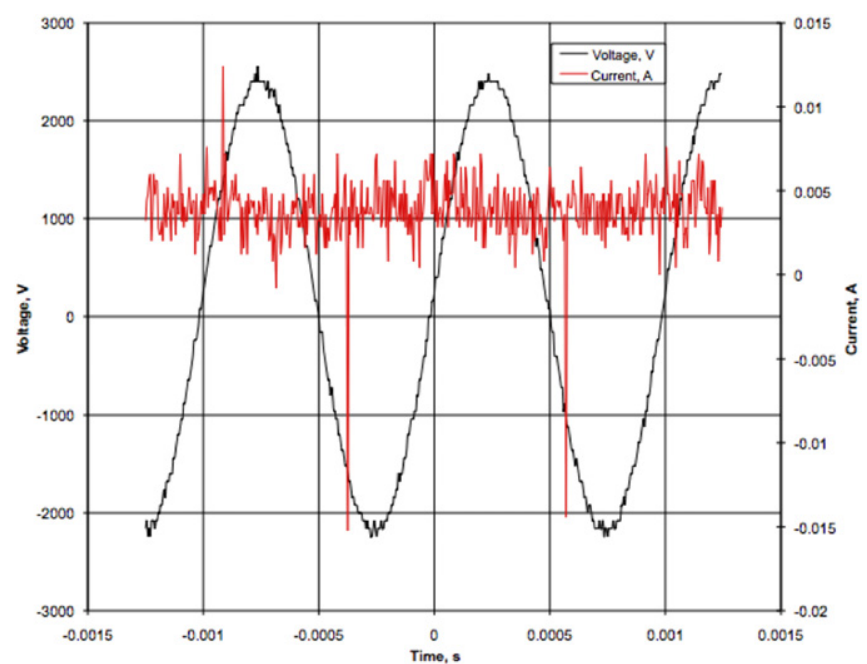

Figure 3. Appearance of microdischarge spikes at initial, low peak-to-peak voltages.

qualitative evolution of the discharge with voltage, (2) the plume heavy particle temperature, determining its dependence on flow and frequency, (3) the physical interaction of the plume with material structures to assess heat transfer and (4) the chemical reactivity induced in water by microdischarge operation alone. This latter task addresses the nature of the chemistry changes in the liquid induced by the plasma. The findings of these experiments are then summarized in the form of a phenomenological model of source operation.

\subsection{Discharge mode evolution: I-V and photographic documentation}

To ascertain the spatial evolution of physical features of air discharge with voltage, particularly within the tube itself, a transparent quartz tube rather than the nominal opaque alumina tube was used. At a fixed flow rate of approximately 1.9 $\mathrm{L} \mathrm{min}^{-1}$, above a threshold peak-to-peak voltage of $4000 \mathrm{~V}$, current spikes associated with the presence of microdischarge formation could be inferred from the appearance of current spikes in the oscillograms [25-27]. As reported earlier and illustrated again in figure 3 , the current spikes appear primarily where the derivative of the voltage (peak-to-peak of $4.6 \mathrm{kV}$ ) with respect to time is the highest. No visible glow could be observed under this condition. At voltages just above $4.6 \mathrm{kV}$, the entire length of the tube glowed visibly albeit faintly to the naked eye as depicted in figure 4, which also shows the associated current-voltage waveform. The glow was diffuse with very little structure. The discharge here behaves simply as a dielectric barrier discharge with both the quartz tube and the liquid water playing the role of dielectric barrier [25]. Further increase in applied voltage gave rise to the appearance of discrete, intense microdischarges amidst a background of purple glow. The transition from diffuse to localized microdischarges is commonly observed in barrier discharges $[28,29]$. In the present case, however, the diffuse glow was present along with localized microdischarges as well, suggesting that multiple modes were present. Such mixed modes have also been observed [30]. In the present case, 


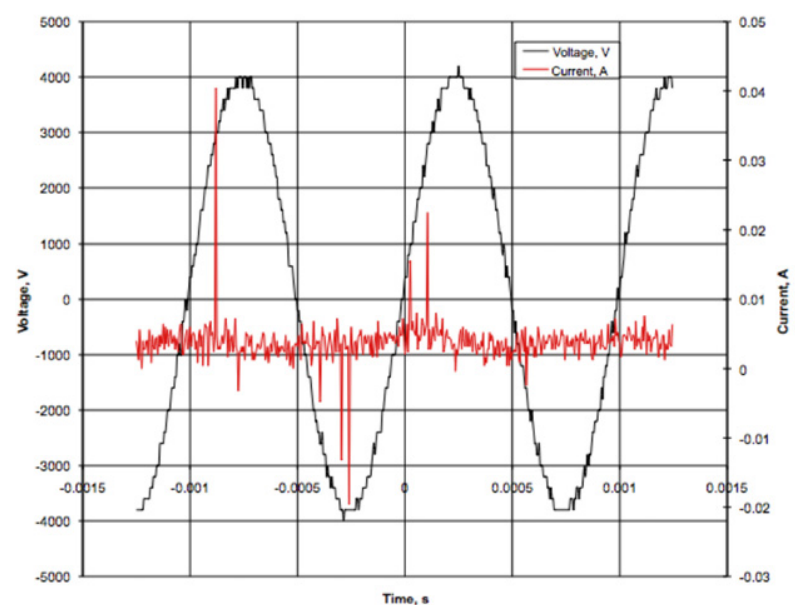

(a)

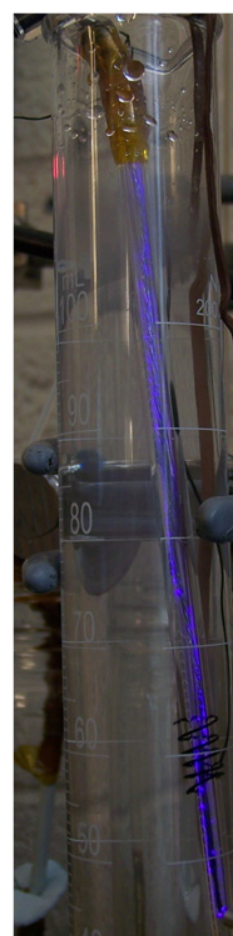

(b)

Figure 4. (a) Discharge waveform and $(b)$ associated photograph of discharge, $9 \mathrm{kV}$ peak to peak.

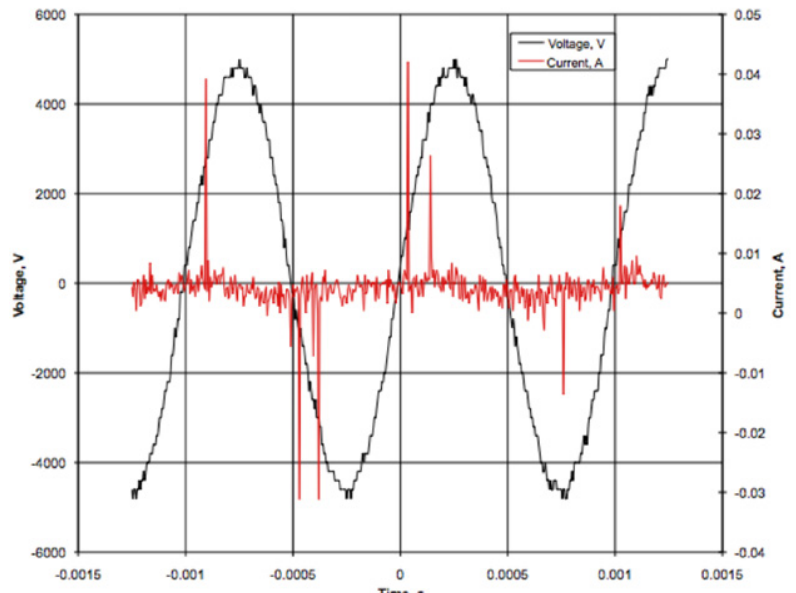

(a)

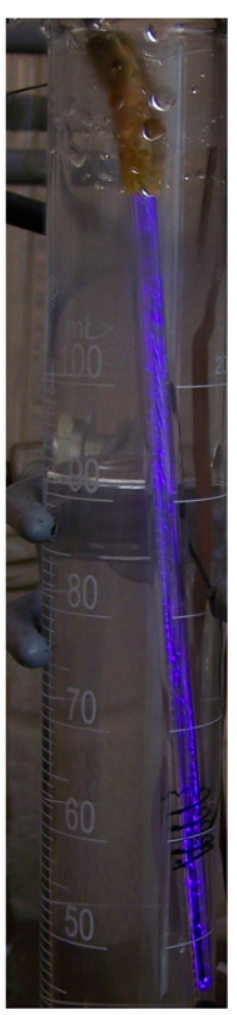

(b)

Figure 5. (a) Discharge waveform and (b) associated photograph of discharge, $9 \mathrm{kV}$ peak to peak.

the intensification of the localized discharges is attributed to localized field intensification at asperities on the surface of the central electrode with increasing voltage. Figure 5 depicts the discrete microdischarges and the diffuse purple background at approximately $9.3 \mathrm{kV}$ peak-to-peak. The oscillogram waveform indicates that both the amplitude and number of the microdischarge current spikes increases considerably with increasing voltage. The photograph indicates that the most 


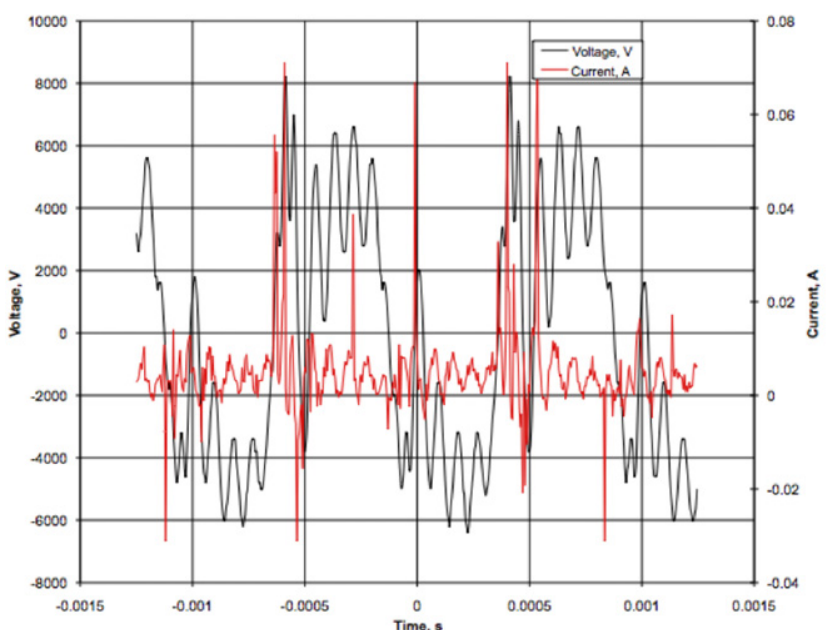

(a)

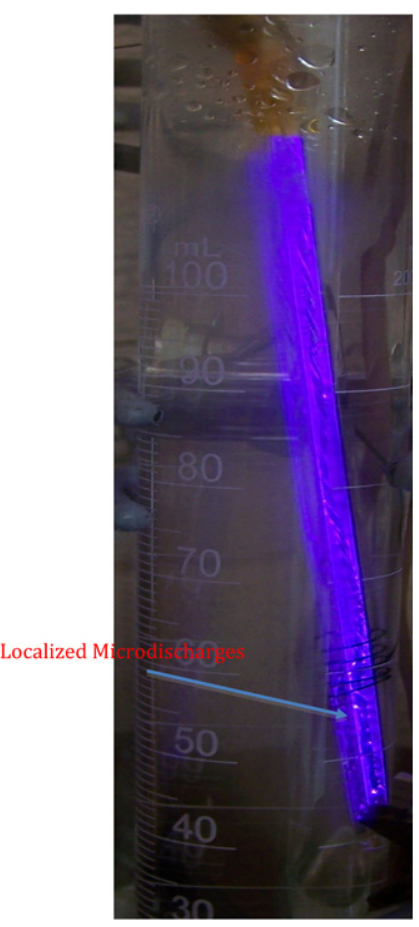

(b)

Figure 6. Sub-plume operation: $(a)$ discharge waveform and $(b)$ discharge appearance.

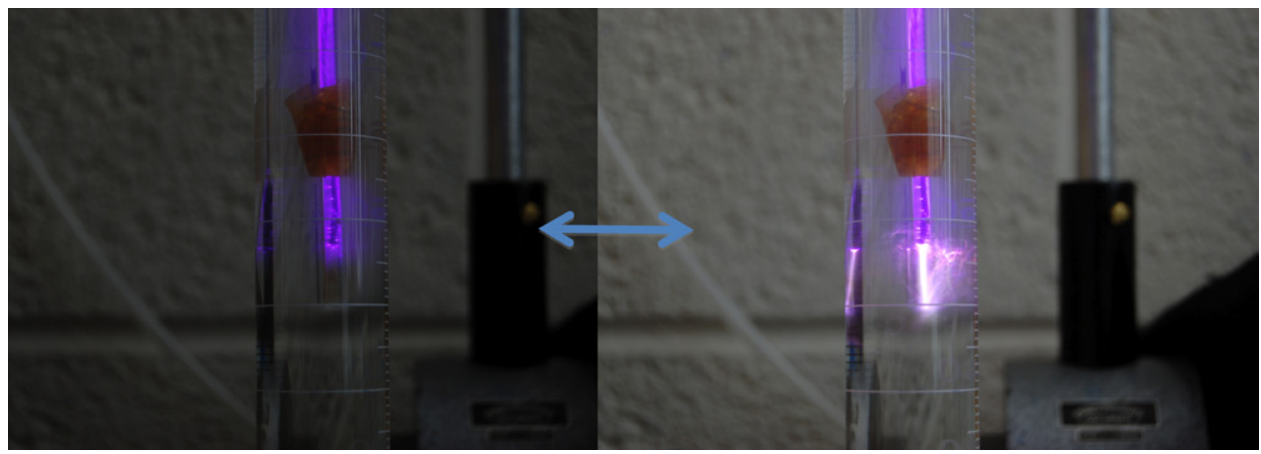

Figure 7. Spontaneous transition into jet mode. Note microdischarge mode also visible.

intense microdischarges occur at the lower end of the electrode. This spatial localization of the microdischarges near the lower end of the electrode becomes more pronounced with increasing voltage. The presence of intense microdischarges in these regions is attributed to the stronger electric field at the electrode tip and the relative closeness of grounded return electrode to this region. Around $12 \mathrm{kV}$ peak-topeak, depicted in figure 6 , the diffuse glow intensifies, as do the very localized microdischarges at the lower end of the tube. The increased discharge brightness is consistent with the increased amplitude in the current spikes. In addition to expected current discharge peaks, an oscillatory, low-level current signal was also observed. The low-level oscillatory structure in the baseline of the $I V$ characteristic is attributed to the power supply/transformer external circuit response to overdriven current spikes. At this voltage level, the discharge was observed to jump between the conditions where a bright diffuse glow superimposed highly localized and bright microdischarges to those in which the microdischarges near the electrode tip were no longer stable or localized. Under this unstable condition, one could clearly observe a streamer-like projection emanating from the end of the powered electrode, alternating termination between points on the adjacent quartz wall and on points along the quartz tube much further downstream. A slight increase in applied voltage further increased the bimodal instability, but in this case arc-like projections actually exited the tube, forming a jet, which emanated from the tip of the electrode and terminated at the water/air pocket boundary. The observed transition between modes is illustrated in figure 7 . The two distinct modes are clearly discernable: (1) diffuse glow with intense microdischarges and (2) diffuse glow with microdischarges and emanating jet. This bimodal behavior transitions into the full plasma jet mode if the voltage is further increased. The full plasma jet mode resulting from a slight increase in voltage is depicted in figure 8 along with the accompanying $I V$ waveform. Once the plume emerges, the peak-to-peak voltage drops considerably from $\sim 12 \mathrm{kV}$ to $7000 \mathrm{~V}$. As can be seen in 


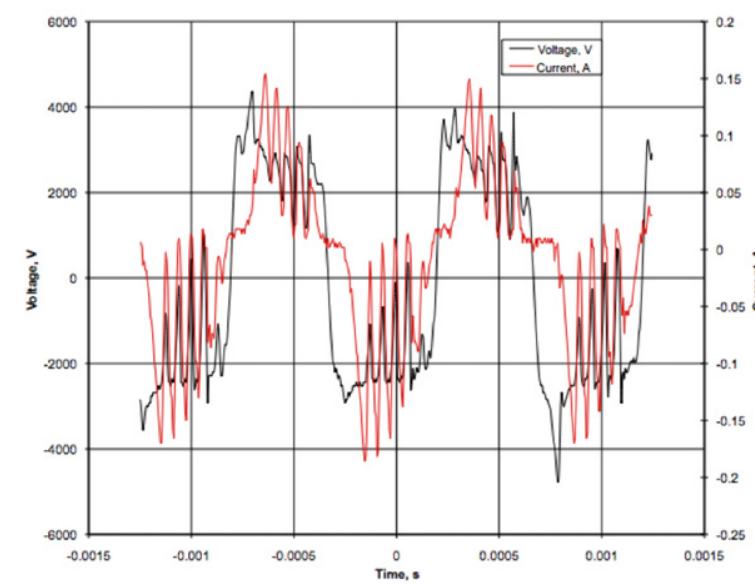

(a)

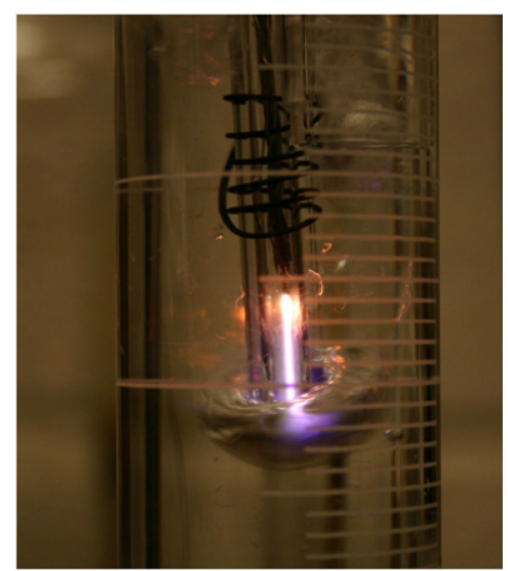

(b)

Figure 8. The emergence of the steady plume mode: $(a)$ waveform and $(b)$ plume mode photograph.

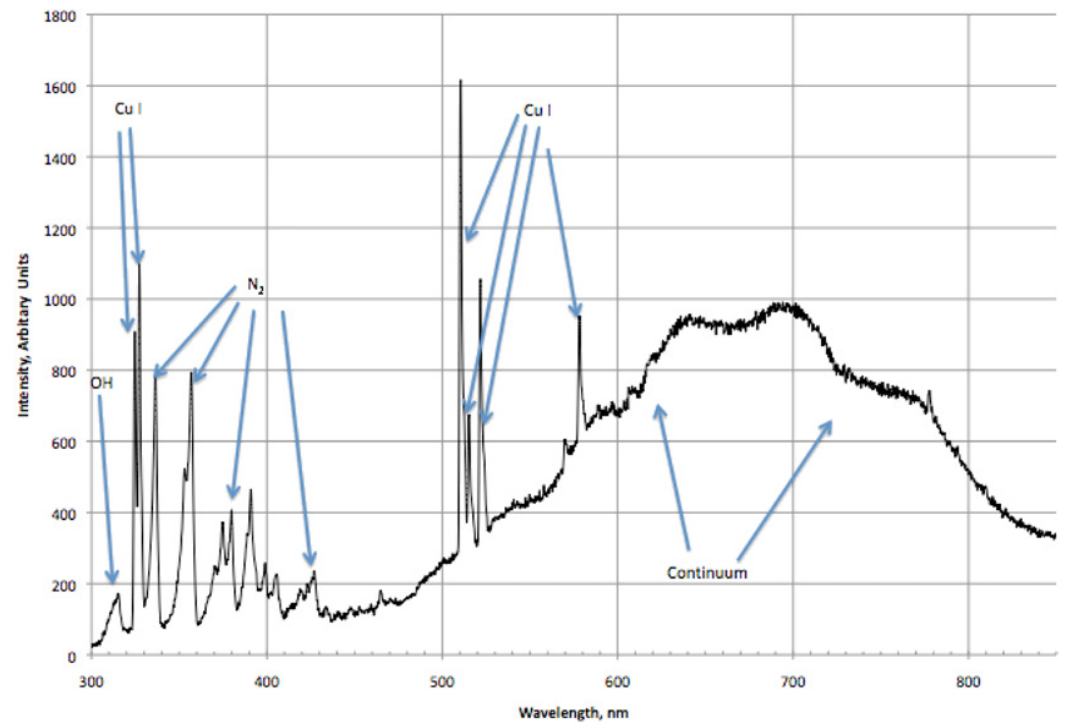

Figure 9. Emission spectra acquired near electrode tip, indicating strong copper lines and continuum emission.

the figure, once the plasma jet emerges, the microdischarges as well as the diffuse glow along the tube are visibly absent. The absence or lack of appreciable microdischarge or tube glow is a direct consequence of the drop in discharge impedance, which once the jet emerges is below the voltage required to first observe the multitude of microdischarges as described earlier (apparent hysteresis). The emergence of the jet mode is associated with audible sound and rapid water heating as previously described [18].

\subsection{Optical emission spectroscopy estimated plasma jet temperature}

Once the discharge transitions into the jet mode, readily noticeable is the white glow located at the tip of the powered electrode. Initially, it was thought that the glow was due to localized heating. In such a case, the spectral intensity profile should be blackbody-like. Typical emission spectra acquired at the electrode tip, shown in figure 9, suggests that the broadband feature was too narrow to be attributed to blackbody radiation. A more likely explanation for the emission appears to be continuum radiation associated with bremsstrahlung and recombination within the dense, electrodederived vapor cloud. Such emission has been observed in electrical machining discharges [31]. If one considers the broad feature to be attributed primarily to bremsstrahlung with the measured peak wavelength of approximately $700 \mathrm{~nm}$, as shown in figure 9, then the associated electron temperature can be estimated: $\lambda_{\max } k_{\mathrm{B}} T_{\mathrm{e}}=h c / 2$, which yields an electron temperature of $0.88 \mathrm{eV}$ [32]. This temperature agrees within $20 \%$ with that estimated from a copper emission Boltzmann plot using the copper 510, 515, 521 and $578 \mathrm{~nm}$ emission lines [31,33].

Further downstream, the continuum radiation is less pronounced, with the spectra instead being dominated by nitrogen, $\mathrm{OH}$ and oxygen emission lines. Copper lines were still present downstream though intensity was considerably lower than the nitrogen emission. High vapor density near the electrode is consistent with cathode spot behavior as discussed later. The measured nitrogen spectra provided a 


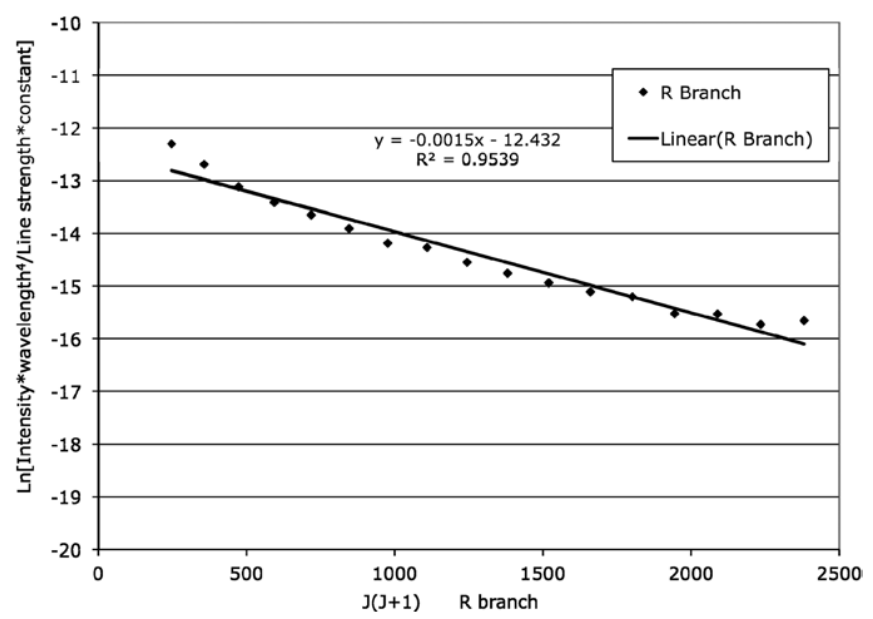

Figure 10. Representative Boltzmann plot of second positive band rotational spectra ( $\mathrm{R}$ branch).

means for diagnosing the gas temperature in the plume. Such temperature information coupled with the estimated electron temperature can be used to determine whether the plasma is nonequilibrium or thermal. The temperature of the jet plume was accessed from unresolved rotational spectra acquired using a spectrometer. In general, the rotational temperature is expected to be representative of the heavy particle temperature. Nitrogen's second positive band corresponding to $C^{3} \Pi_{\mathrm{u}} \rightarrow$ $B^{3} \Pi_{\mathrm{g}}$ was used to estimate the temperature of the gas in the plume. In particular, the $\Delta v=0$ sequence was fitted to obtain the rotational temperature following Hopwood and others [34-36]. This sequence has strong emission around $337 \mathrm{~nm}$. The temperature can be obtained by plotting the natural logarithm of the intensity versus the rotational quantum number of the second positive band:

$$
\operatorname{Ln}\left|\frac{I_{j^{\prime}-j^{\prime \prime}} \cdot \lambda^{4}}{c \cdot S_{j^{\prime}-j^{\prime \prime}}}\right|=\frac{-\left(\beta_{\mathrm{e}}-\alpha_{\mathrm{e}}\left(v^{\prime}+\frac{1}{2}\right)\right) \cdot j \cdot(j+1) \cdot h c}{k T_{\text {rot }}}
$$

where $\beta_{\mathrm{e}}$ is the rotational term corresponding to the equilibrium bond length, $\alpha_{\mathrm{e}}$ is a positive constant associated with the vibrational term, $I_{j^{\prime}-j^{\prime \prime}}$ is the line intensity, $S_{j^{\prime}-j^{\prime \prime}}$ is the line strength and $T_{\text {rot }}$ is the rotational temperature. Equation (1) follows from the proportionality between the line intensity and the product of upper state population, which is described by a Boltzmann distribution, the line strength and the frequency to the fourth power [37]. Because the upper states are described by a Boltzmann distribution, the closely spaced rotational lines can be approximated as a continuous emission profile and are measured as such at low resolution. This profile is directly measured by the spectrometer. Constants and line strengths were obtained from Hertzberg [38]. The left-hand side of equation (1), acquired from measured spectra, was plotted versus the rotational quantum number to obtain the Boltzmann plot. As shown in figure 10 which illustrates representative data, acquired second positive band spectra around $337 \mathrm{~nm}$ appear to be reasonably well described by the Boltzmann distribution, allowing for an estimate of the rotational temperature to be made. The calculated spectra also agreed well with the Specair band analysis software [39]. In
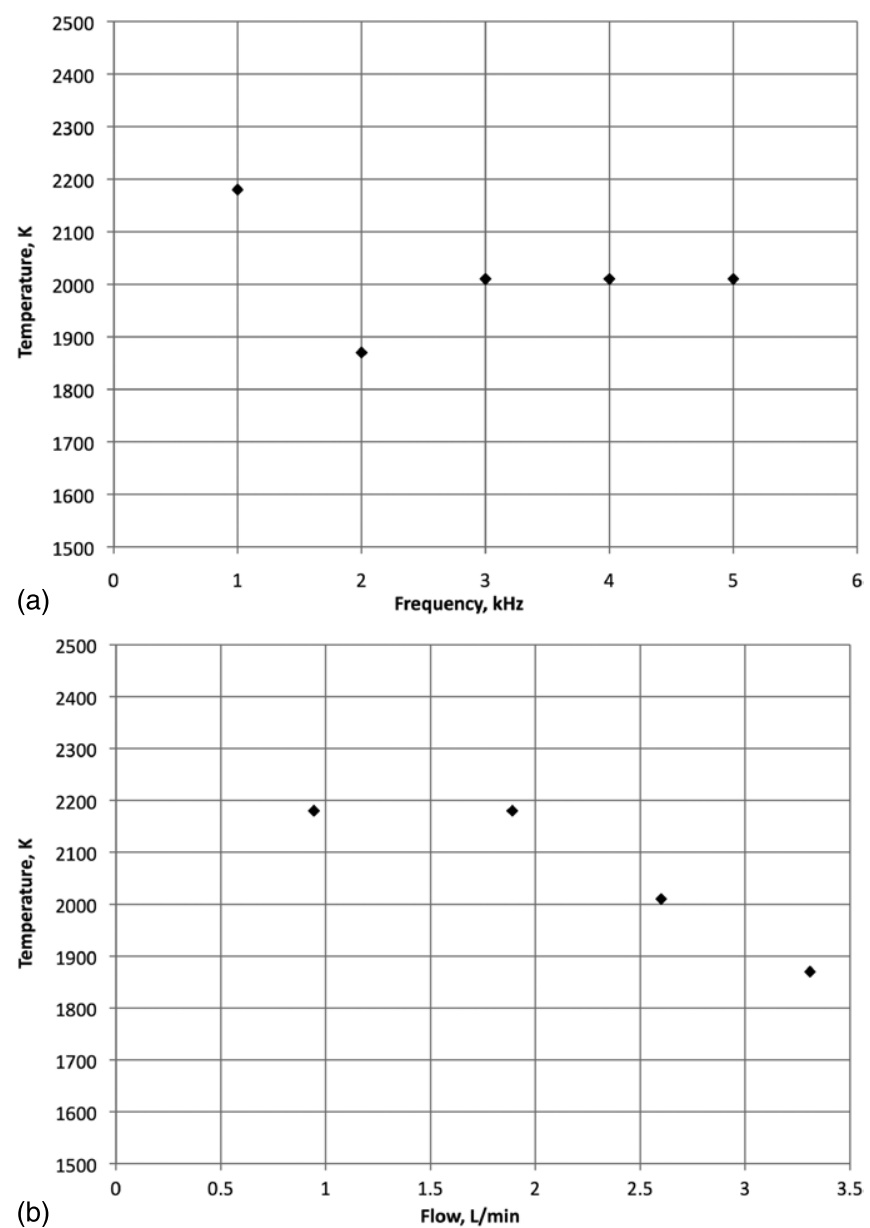

Figure 11. Variation in heavy particle temperature with (a) frequency and $(b)$ flow rate at $1 \mathrm{kHz}$.

this manner, the variation in the gas temperature of the plume was determined both as a function of frequency and of flow rate. The peak-to-peak voltage in the jet mode, which did not vary appreciably with frequency, was on average $3 \mathrm{kV}$ for the operating conditions investigated where the input airflow rate was set at $1.4 \mathrm{~L} \mathrm{~min}^{-1}$. The variation of temperature with frequency is presented in figure 11. Over the frequency range investigated, the temperature did not vary appreciably. A small drop of $10 \%$ was observed as frequency was increased from 1 to $2 \mathrm{kHz}$. Beyond $2 \mathrm{kHz}$ the temperature increased slightly, saturating at approximately $2000 \mathrm{~K}$. The slight drop from 1 to $2 \mathrm{kHz}$ may be due to the associated peak-to-peak voltage drop of approximately $13 \%$. The invariance of temperature with frequency was consistent with the constant water heating rate and power deposition observed [18].

The magnitude of the heavy particle temperature suggests significant heavy particle heating. Such arc-like temperatures have been observed in low frequency air-water and high pressure dc microdischarge sources [40,41]. Consistent with inferred optical emission spectroscopy temperature was observed damage to a downstream, test aluminum substrate and the accompanying aluminum vapor. The boiling point of aluminum is $2792 \mathrm{~K}$. Such high temperatures are possible only if there is a sufficiently high collision frequency between the electrons and the neutrals $[41,42]$. The hot gas which in 


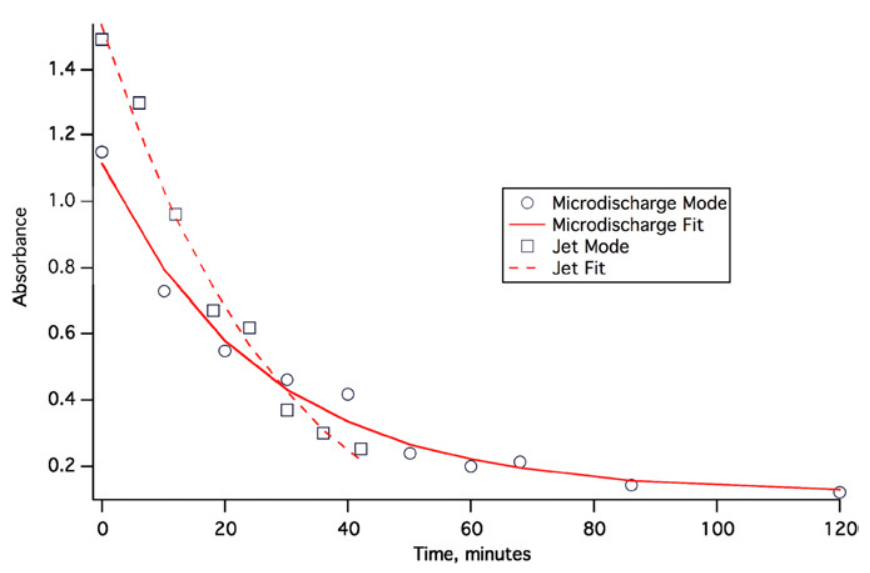

Figure 12. Comparison between jet mode and microdischarge decomposition rates.

turn transfers its thermal energy into the water is the primary origin of the rapid water heating during jet mode operation. It should also be pointed out that the density of air at these temperatures is nearly an order of magnitude lower than room temperature air at similar pressures. The associated $E / N$ therefore is significantly increased, giving rise to increased ionization.

The flow rate was varied from 0.9 to $3.3 \mathrm{~L} \mathrm{~min}^{-1}$ to assess jet sensitivity to gas flow rate at a fixed frequency of $1 \mathrm{kHz}$. Although the peak-to-peak voltage increased from $3.5 \mathrm{kV}$ peak-to-peak to nearly $6 \mathrm{kV}$ peak-to-peak, the measured temperature actually decreased with increasing flow rate as illustrated in figure 12 by about $15 \%$. Such convective cooling was expected with increasing gas flow. Such temperature reduction with increasing flow rate has been observed elsewhere [40]. Interestingly, with increasing flow rate, the intensity of the copper emission decreases markedly. Indeed beyond $2 \mathrm{~L} \mathrm{~min}^{-1}$ only the copper $521 \mathrm{~nm}$ line is indistinguishable from the baseline. This observation appears to suggest that gas dynamic cooling plays a key role in discharge behavior. The reduction in intensity is most likely due to increased forced air-cooling of the electrode tip. This observation is consistent with the measured decreases in peak discharge current (both negative and positive peaks) with increasing flow for those flow-rates over $2 \mathrm{~L} \mathrm{~min}^{-1}$. This cooling would explain why an increased voltage is required for the jet mode at the higher flow rates.

\subsection{Comparative chemical reactivity study}

It was shown in an earlier study that the jet mode operation introduces significant chemical reactivity into the water [18]. In that study, a MB dye solution was decolored upon exposure to the plasma jet. Accompanying the decoloration was a decrease in solution $\mathrm{pH}$. The decoloration is attributed to advanced oxidation of the dye driven by the presence of the $\mathrm{OH}$, ozone and atomic oxygen radicals. The $\mathrm{pH}$ reduction is attributed to the formation of acidic decomposition products [43]. As has been pointed out in other studies, MB can be decomposed in strongly oxidizing environments, leading to clearing [44-48]. Indeed, the use of pulsed discharges to treat dye-laden water is based on the mechanism of decomposition of MB by oxidation. Additionally, associated with the high temperatures in the plasma jet, it is to be expected that some plasma pyrolysis of MB also occurs [49]. Indeed, it may be that the water at the plasma jet-water interface is indeed supercritical, at least locally.

Because of the apparent importance of oxygen radicals, the question that naturally arises is whether the higher temperature jet mode is necessary to induce the chemical reactivity. We have shown that the discharge evolves from an intense microdischarge to the unterminated jet. During such processing, is the decoloration of the dye due to the formation of oxidizers in the gas-phase discharge, the air-water interface, or a combination of both? If gas-phase derived precursors play an important role, then operating in the microdischarge mode (discharge resides in tube only) should be sufficient to clear the dye. In this case, microdischarge-derived plasma products can be expected to enter the liquid as they diffuse out of the discharge tube into the MB solution.

A $6 \mathrm{mg} \mathrm{L}^{-1} \mathrm{MB}$ solution in deionized water was treated with the microdischarge mode to assess its capacity for decolorizing. During the plasma exposure, the MB fluid was batch processed using a small pump, which continuously circulated the solution at a flow rate of $9 \mathrm{ml} \mathrm{min}^{-1}$ from the $400 \mathrm{ml}$ solution reservoir, through a flask containing the discharge tube and back again. Processing was carried out in a closed loop for $2 \mathrm{~h}$. Figure 12 depicts the time evolution of the MB solution absorption using a spectrophotometer following procedures described in detail in [18], which indicated comparable effectiveness. For comparison purposes, data from an earlier jet mode experiment in which a $7 \mathrm{mg} \mathrm{L}^{-1}$ solution of MB was treated in a similar manner are also plotted alongside the microdischarge-only data. The jet mode time constant was approximately $20 \mathrm{~min}$ in contrast to the microdischarge time constant of approximately $36 \mathrm{~min}$. The time constants refer to the characteristic decolorization time for $400 \mathrm{ml}$ of the MB solution. The advantage of the microdischarge mode is the associated low solution processing temperature, which remained at room temperature during the entire processing run in contrast to the nominal $70^{\circ} \mathrm{C}$ solution temperature for the jet mode. Process time, however, is sacrificed when compared with the jet mode.

\section{Discussion}

A cursory glance at the $I V$ characteristic (see figure 8) associated with the jet mode would lead one to conclude that the shape of the discharge current signal is relatively independent of the voltage polarity. Careful inspection of the waveform, however, reveals a significant difference. During the negative portion of cycle, the current consists of essentially a series of pulses. The pulses essentially turn on at sufficiently high negative voltage, peak and then extinguish with the current level dropping rapidly through zero with some overshoot. On the other hand, during the positive cycle, the discharge current also consists of a series of pulses but in this case the pulses are superimposed upon a background current, presumably the background afterglow. The pulses on the 
positive cycle occur regularly but they do not pass through zero. In the positive applied voltage case, it is conjectured that the discharge consists of intermittent streamer-driven filamentation coexisting with a microdischarge remnant [50]. The diffuse plasma is attributed to afterglow from positive streamers. The background afterglow can survive between pulses if the pulse frequency is sufficiently high. In this work, the frequency of occurrence of current spikes during the positive cycle is $20 \mathrm{kHz}$. Remnant lifetimes are of the order of milliseconds. This effect is enhanced by the fact voltage does not return to zero before the next filament restarts. The simultaneous appearance of background remnant and microdischarge has been observed elsewhere [51]. So once the microdischarge is struck, charging of the dielectric forces the voltage below the threshold value to support the Townsend discharge causing the microdischarge to go out. But the discharge remnant can still participate in the current transport if the remnant density is sufficiently high. Negative ion constituents of the remnant would drift toward the positively biased electrode at a rate determined by the time varying density and the mobility. In this regard, it is conjectured that it is this remnant current flow that maintains a baseline current between pulses-preventing the current from going to zero. Interestingly, on the positive cycle, the amplitude of the current pulses decrease with increasing time, following applied voltage until the voltage drops below ignition value. During the negative cycle, the discharge is characterized by distinct, current pulses that decay to essentially zero over a microseconds time scale, reminiscent of cathode spots [52]. A cathode spot discharge essentially burns itself out as the localized discharge at the electrode grows spatially in size to the point that localized power deposition is no longer sufficient to maintain electron and vapor emission. The cathode spotlike behavior would explain why the discharge goes out over microsecond time scales. The absence of remnant background plasma between cathode spot pulses can be attributed to the high plasma flow speeds coupled with the fact that cathode spot maintenance depends on local electrode processes and not gas-phase plasma production. Indeed for flows $<2.5 \mathrm{~L} \mathrm{~min}^{-1}$, current waveforms were essentially independent of flow rate. Positive current waveforms, on the other hand, were more sensitive to flow rate, essentially decreasing in amplitude with increasing flow rate. When the cathode spot extinguishes, the discharge essentially goes out. The voltage near the end of the negative current pulse also drops to nearly zero. Such low fields cannot be expected to maintain a background, diffuse plasma as in the case of the discharge in the positive cycle. It should also be pointed out that independent of voltage polarity, the current pulses do not appear over the entire negative cycle, rather they are bunched in time, essentially occupying those time periods where the voltage is sufficiently high, which accounts for about $50 \%$ of the time during a half cycle. Taken together, these data suggest that the discharge is arc-like during the negative cycle with the plasma properties driven presumably by cathode spot phenomena at the electrode surface and microdischarge dominated during the positive cycle.

Anecdotal evidence of this bimodal, polarity dependent behavior is also suggested by fast camera images acquired at the tip of the discharge electrode, presented in figure 13. The tip was imaged at 5000 frames s$^{-1}$. At this rate, one can sample evolution of the discharge as a function of time over the cycle- 5 snapshots per cycle. In general, it was observed that the jet-like phenomena tended to occur half the time with a dimmer, more diffuse glow occurring during other portions of the cycle. These visual observations are consistent with the implications suggested by the waveforms: half the cycle discharge is a diffuse barrier discharge with intermittent filamentation while the other portion of the cycle consists of cathode spot-like phenomena. The plasma jet originated at localized points on the surface of the powered electrode. Indeed, the source of the jet on the surface of the electrode tended to move around the surface, just as one would expect of a conventional cathode spot. It was also observed that just before the jet mode, filamentation of a localized discharge between the electrode and the tubing was observed. Presumably, such localization makes conditions favorable for subsequent cathode spot formation after the electrode goes negative. Observed powered electrode surface texturing, signs of melting, and the presence of copper in the emission spectra clearly indicate electrode processes are active during discharge operation, providing further evidence of cathode spot-like phenomena.

The question of what happens to the deposited electron charge during the negative cycle of the jet mode naturally arises. Water in general has excellent dielectric properties with a dielectric constant of 80 for deionized water. But under electron irradiation, a number of reactions can take place with the water molecules: direct ionization requiring $2.6 \mathrm{eV} \mathrm{mol}^{-1}$ :

$$
\mathrm{H}_{2} \mathrm{O} \rightarrow \mathrm{H}_{2}+\frac{1}{2} \mathrm{O}_{2}
$$

or disassociative attachment:

$$
\begin{gathered}
\mathrm{e}+\mathrm{H}_{2} \mathrm{O} \rightarrow \mathrm{H}^{-}+\mathrm{OH} \\
\mathrm{H}^{-}+\mathrm{H}_{2} \mathrm{O}^{+} \rightarrow \mathrm{H}_{2}+\mathrm{OH}^{-} .
\end{gathered}
$$

Each of these processes provides pathways for electron transport off the water-air interface. In this regard, the water essentially behaves as a leaky capacitor. This in general suggests that water can support a larger charge transfer in comparison with a solid dielectric since the charge buildup must compete with disassociative attachment and diffusion processes. Such effects would also limit the maximum current pulse amplitude, preventing current runaway to an actual arc discharge [53].

\section{Concluding remarks}

Discharge evolution and plasma jet plume characteristics have been measured. Data suggest that the discharge is bimodal in nature with microdischarge processes dominating during the positive cycle and arc-like processes occurring during the negative cycle. This biomodal nature suggests that by tailoring the duration of the negative and positive pulses of the discharge, the diffuse barrier discharge or arc-like character 


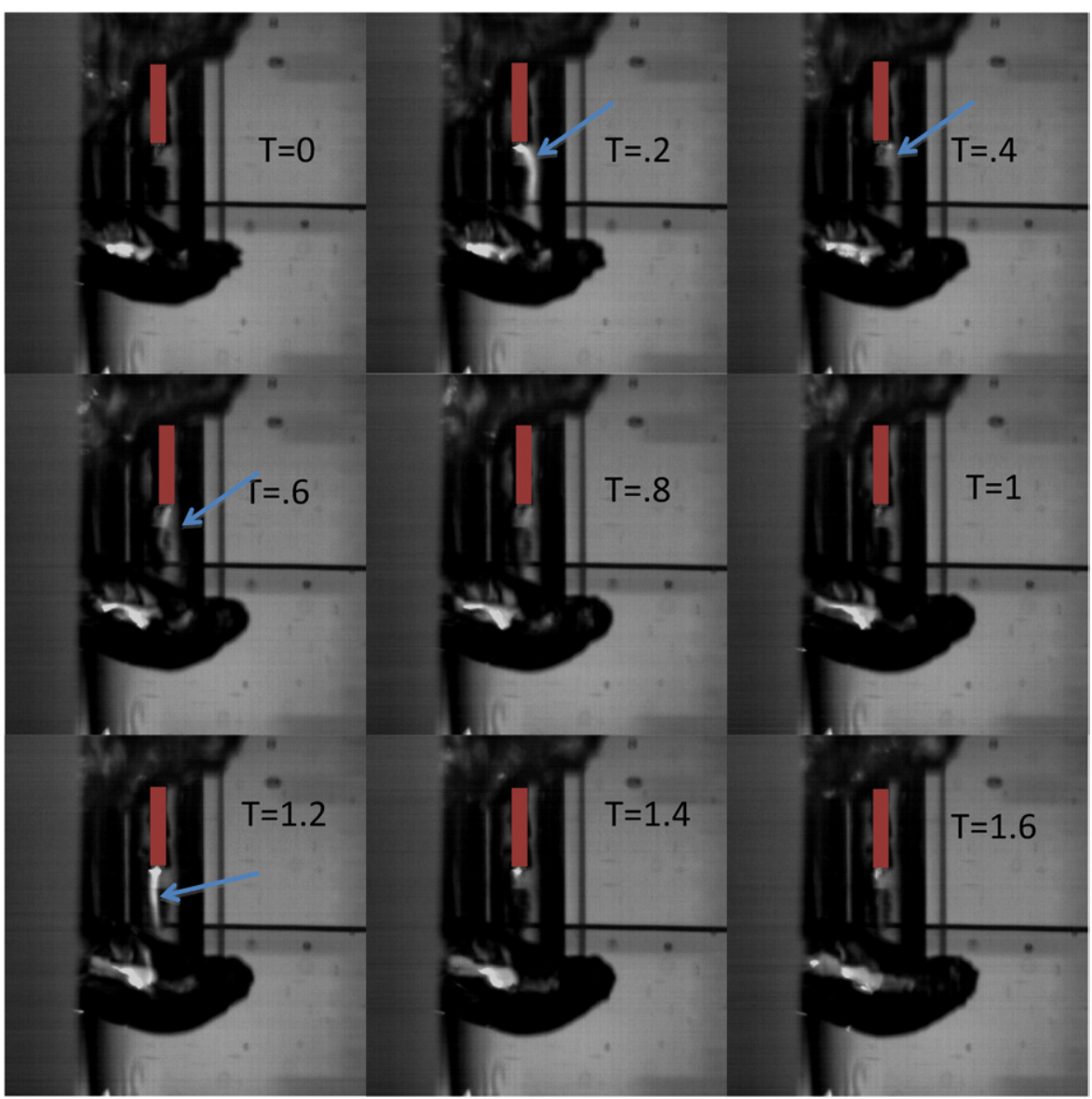

Figure 13. Discharge evolution over a cycle. Time period between frames, $0.2 \mathrm{~ms}$. Note jet appears only once per cycle.

can be tailored. Such tailoring could be used to tailor chemical reactivity induced by the discharge. Such control could also allow for the adjustment of average degree of equilibrium that exists between electrons and heavy species over a cycle. Rotational spectral measurement indicated jet temperatures of nearly $0.2 \mathrm{eV}$. The utility of such a high temperature plasma has been pointed out by Graves as means to achieving a chemical reaction medium for certain endothermic reactions [54]. It should also be pointed out that the bimodal nature of the discharge suggests new opportunities for processing. For example, processing requiring low temperature activation and subsequent high temperature annealing or activation energy is conceivable with the DBD plasma jet.

\section{Acknowledgments}

This work was supported by the National Science Foundation (CBET 0939879) and the NRC (38-08-949 Faculty Development Grant). The authors would also like to thank Laura Gallagher for temperature calculations with Specair [39] and Cameron Howard for acquiring decolorization data in the microdischarge mode.

\section{References}

[1] Grabowski L R et al 2007 Plasma Sources Sci. Technol. 16226

[2] Locke B R et al 2006 Indust. Eng. Res. 45882
[3] Malik M A, Ghaffar A and Malik S A 2001 Plasma Sources Sci. Technol. 1082

[4] Gao J-z, Wang X-y, Hu Z-a, Hou J-g and Lu Q-f 2001 Plasma Sci. Technol. 3765

[5] Klapkiv M, Nykyforchyn H and Pousvailo V 1994 Mater. Sci. 30

[6] Anpilov A et al 2001 J. Phys. D: Appl. Phys. 34993

[7] Kravchenko A, Berlizova S, Nesterenko A and Kublanovskii V 2004 High Energy Chem. 38333

[8] Glaze W, Kang J and Chapin D 1987 Ozone Sci. Eng. 9335

[9] Sugiarto A, Ohshima T and Sato M 2002 Thin Solid Films 407174

[10] Bruggeman P, Leys C and Vierendeels J 2006 J. Appl. Phys. 99116101

[11] Katsuki S, Akiyama H, Ghazala A and Schoenbach K H 2002 IEEE. Trans. Dielectr. Insul. 9498

[12] Sugiarto A, Ohshima T and Sato M 2002 Thin Solid Films 407174

[13] Akiyama H 2000 IEEE. Trans. Dielectr. Electr. Insul. 7646

[14] Korobeinikov S W, Melekhov A V and Besov A S 2002 High Temp. 40652

[15] Grabowski L, Veldhuizen E, Pemen A and Rutgers W 2007 Plasma Sources Sci. Technol. 16226

[16] Yamabe C, Takeshita F, Miichi T, Hayashi N and Ihara S 2005 Plasma Process. Polym. 2246

[17] Ishijima T, Sugiura H, Saito R, Toyoda H and Sugai H 2010 Plasma Sources Sci. Technol. 19015010

[18] Foster J, Weatherford B, Gillman E and Yee B 2010 Plasma Sources Sci. Technol. 19025001

[19] Grabowski L, Veldhuizen E, Pemen A and Rutgers W 2007 Plasma Sources Sci. Technol. 16226 
[20] Kuo W G 1992 Water Res. 267881

[21] Pavan F, Mazzacato A and Gushikem Y 2008 Bioresource Technol. 993165

[22] Spear M 2006 R\&D takes the fast track Chemical Processing.com, http://www.chemicalprocessing. com/articles/2006/143.html

[23] Babaeva N and Kushner M J 2009 J. Phys. D: Appl. Phys. 42132003

[24] Piskarev I 1999 Tech. Phys. 4453

[25] Kogelschatz U 2003 Plasma Chem. Plasma Process. 231

[26] Hoder T, Sira M, Kozlov K V and Wagner H-E 2008 J. Phys. D: Appl. Phys. 41035212

[27] Kang W, Kim Y, Hong S and Song Y 2002 Numerical modeling on recurrence of microdischarges in dielectric barrier discharge Proc. 8th Symp. on High Pressure, Low Temperature Plasma Chemistry (Puhajarve, Estonia) (Hakone VIII)

[28] Muller I, Punset C, Ammelt E, Purwins H-G and Boeuf J P 1999 IEEE Trans. Plasma Science 2720

[29] Kundhardt E E 2000 IEEE Trans. Plasma Sci. 28498

[30] Petit M, Goldman A and Goldman M 2002 J. Phys. D: Appl. Phys. 352969

[31] Descoeudres A 2005 J. Mater. Proc. Technol. 149184

[32] Kunze H 2009 Introduction to Plasma Spectroscopy (New York: Springer)

[33] Li B and Li H 2001 Discussion on emission spectroscopy measurements from dense electrothermal launcher plasma Proc. 19th Int. Symp. of Ballistics (Interlaken, Switzerland, 7-11 May 2001)

[34] Iza F and Hopwood J A 2004 IEEE Trans. Plasma Sci. 32498

[35] Sarani A, Nikiforov A Y and Leys C 2010 Phys. Plasmas 17063504

[36] Valdivia-Barrientos R et al 2008 Laser Phys. 18298

[37] Marr, G 1968 Plasma Spectroscopy (Amsterdam: Elsevier)

[38] Hertzberg G and Spinks J 1950 Molecular Spectra and Molecular Structure (New York: Van Nostrand-Reinhold)
[39] Laux C O, Gessman R J and Kruger C H 2002 Measurements and modelling of the absolute spectral emission of air plasmas between 185 and $800 \mathrm{~nm}$, in preparation

Laux C O 2002 Radiation and nonequilibrium collisional-radiative models von Karman Institute Special Course on Physico-Chemical Modeling of High Enthalpy and Plasma Flows (Rhode-Saint Genese, Belgium, 4-7 June)

[40] Park J Y, Kostyuk P, Hans S, Kim J and Lee H 2006 J. Phys. D.: Appl. Phys. 393805

[41] Penache C et al 2002 Plasma Sources Sci. Technol. 11476

[42] Kushner M J 2005 J. Phys. D. Appl. Phys. 381633

[43] Sugiarto A, Ohshima T and Sato M 2002 Thin Solid Films 407174

[44] Sano J, Fujikawa J, Kawashima T, Kanki T and Toyoda A 2002 Decomposition treatment of dye solutions using direct contact gas corona discharge J. Japan Soc. Water Environ. 25 763-2065

[45] Malik M 2003 Plasma Sources Sci. Technol. 12 S26

[46] Magureanu M, Mandache N and Parvulescu V 2007 Plasma Chem. Plasma Process. 58927

[47] Magureanu M et al 2008 Plasma Chem. Plasma Process. 28677

[48] Grabowski L R 2007 Plasma Sources Sci. Technol. 16226

[49] Willberg D M et al 1996 Environ. Sci. Technol. 302526

[50] Fridman, A 2008 Plasma Chemistry (Cambridge: Cambridge University Press)

[51] Petit M, Goldman A and Goldman M 2002 J. Phys. D: Appl. Phys. 252969

[52] Boxman R, Sanders D and Martin P 1995 Handbook of Vacuum Arc Science and Technology (with forward by J Lafferty) (Park Ridge, NJ: Noyes Publication)

[53] Bertault P, Dupuy J and Gilbert A 1977 J. Phys. D: Appl. Phys. 10 L219

[54] Hsu D D and Graves D B 2003 J. Phys. D: Appl. Phys. 362898 